\title{
Information processing in an optoelectronic display system
}

\author{
A.V. Bushma \\ Odessa National Academy of Telecommunications named after O.S.Popov \\ 1, Kuznechnaya str., 65029 Odessa, Ukraine
}

\begin{abstract}
Generalized analytical description of a display system based on the functional approach to data processing in the human-machine environment has been made. Logic properties of a separate display element have been investigated. It has been shown that polar elements realize the logical function of negation of implication and non-polar ones process the data according to the inequality function. Equivalent circuits in the Boolean basis that can replace polar and non-polar information area elements in logical functional modeling of display units have been presented.
\end{abstract}

Keywords: data processing, digital display, information model, logical operator, logic gate.

Manuscript received 26.01.10; accepted for publication 16.03.11; published online 30.06.11.

\section{Introduction}

Usage of the system approach for investigation and optimization of the functional and hardware realization of optoelectronic human-machine means ensures extra efficiency of the analysis and demanded level of reliability of the obtained results. The information display system (IDS) is here the key element defining quality indexes of all the hierarchy of technical designs. Thus, at various levels of generalization of their implementation the most effective is informational representation of an optoelectronic system for data transfer to an operator that corresponds to its place and a role in higher levels of system hierarchy. Description formalization of information processing will allow to simplify investigation of structural parameters of complex optoelectronic devices and to attain a necessary level of generalization of the obtained results.

High reliability of interaction between the operator and technical means is provided with use of optoelectronic displays in systems and devices, that are the most effective for different functions from the ergonomic viewpoint [1]. Such constructions of ergatic systems assume corresponding functional and design features in their configuration. It is obvious that in any structure the display device carries out the function of transformation of an entrance information signal to the visual one that is intended to the operator. Therefore, use of general analytical representations that are based on principles of information transform for research and the analysis will allow to provide the uniform methodological approach to the description of IDS hardware decisions. As a result, creation of technical realizations and their criterial optimization becomes simpler. However, these analytical approaches are developed insufficiently.

The analysis and analytical interpretation of information processing in the ergatic optoelectronic system and its units has been represented in this work.

\section{Formalized presentation of information processing}

Generally, optoelectronic IDS can be presented in the form of the abstract converter of information. To its input, the stream of messages $F_{0}(t)$ arrives from an external system for data processing. At the exit, with a delay $\Delta t$ the stream $F_{\mathrm{S}}(t+\Delta t)$ that is intended for registration by the visual analyzer of the person-operator is formed. The stream $F_{\mathrm{S}}(t+\Delta t)$ is created of symbols $S_{v}$ that in the visual form present data upon controllable information [2]. The set of used symbols is defined by an information model (IM) and forms its alphabet $\Omega_{\mathrm{IM}}$, which is described by the following set

$\Omega_{\mathrm{IM}}=\left\{S_{1}, S_{2}, \ldots, S_{v}, \ldots, S_{(l-1)}, S_{l}\right\}$,

where $S_{v}$ is the $v$ - th symbol (element), and $v=\overline{1, l}$;

$l$ is the length of the IM alphabet.

The number of IM elements corresponding to the length of alphabet $l$, is the foundation of the code of the 
alphabet $\Omega_{\mathrm{IM}}$ (1). To form the visual images implementing IM, the part of space representing information area (IA) is used. The image synthesis is carried out at IA by excitation of its elements (IAE).

In the optoelectronic IDS, the physical nature of input and output signals essentially differs. Realized in them is conversion of a data stream $F_{0}(t)$ possessing, as a rule, the electrical form, in the optical spatial-andtemporal stream $F_{\mathrm{S}}(t+\Delta t)$. Its parameters are defined by IM of message presentation in the system and provide the visual alphabet of characters, which is implemented on set of elements

$\boldsymbol{A}=\left\{a_{1}, a_{2}, \ldots, a_{i}, \ldots, a_{p-1}, a_{p}\right\}$,

where $\boldsymbol{A}$ is IAE set; $a_{i}$ is the $i$-th element, and $i=\overline{1, p}$; $\mathrm{p}$ - total amount of IAE.

It is obvious that IDS at the moment of time $t$ processes the input data according to the equation

$\psi\left[F_{0}(t)\right]=F_{\mathrm{S}}(t+\Delta t)$,

where $\psi$ is the operator describing the process of information transform in the system.

Data in IDS are processed sequentially in three stages. This process described by the expression (3) is implemented by three functional units that can be presented as

$\Psi=\left\{\psi_{i}\right\}$,

where $\psi_{i}$ is the conversion operator, $i=\overline{1,3}$, and, $\psi_{1}$ is the operator corresponding to process of data recording; $\psi_{2}$ - operator reflecting data storage; $\psi_{3}$ - operator corresponding to data output.

As a result of action of operators $\psi_{i}$, the input sequence of messages $F_{0}(t)$ is transformed to appropriate symbols $S_{\mid}$. In IDS rigidly determined algorithm of operation of the defined IM is realized. Thus, the main functional loading defined by specificity of system and IM, lays down on structure elements that carry out data conversion according to the operator $\psi_{3}$. It is fulfilled according to IM and described by the set of operators

$\Psi_{3}=\left\{\Psi_{3} j\right\}$,

where $\psi_{3 j}$ is the $j$ - th conversion operator; $j=\overline{1,4}$, and, $\psi_{31}$ is the recoding operator; $\psi_{32}$ - operator describing conversion of signals for the coordination of electrical parameters of the code and exciting influence for an electrooptical transformer (EOT); $\psi_{33}$ conversion operator of an electric signal into the optical one; $\psi_{34}$ - operator corresponding to optical handling the image.

In real information systems, the input data stream $F_{0}(t)$ is sequence of the codes $C_{v}$, each of which corresponds to the fixed output message $S_{v}$ from the alphabet $\Omega_{\mathrm{IM}}$. In general, input and output streams for IDS are asynchronous, and the necessary sequence of conversions is described by the sets of operators (4) and (5). Thus, synthesis of visual symbols $S_{v}$ is usually carried out cyclically in a dynamic mode. The appropriate period of image refresh is equal $T_{S}$. Then formation of the arbitrary $v$-th message $S_{v}$ from the input code $C_{v}$, proceeding from expression (3) and with account of (4) and (5) for time $t$, presents situation that can be obtained in the following aspect

$S_{v}=\left.\psi_{33}\left\{\psi_{32}\left\{\psi_{31}\left\{\psi_{2}\left\{\psi_{1}\left(C_{v}^{I}\right)\right\}\right\}\right\}\right\}\right|_{t=t+0} ^{t=t+T_{S}}$.

This expression is a basis for the formalized description of signal processing in IDS. Analytical representation for each of the used operators corresponding to separate stages of the process for information transform, their analysis and mutual minimization allow optimizing hardware solutions at the function level.

\section{Display as an information transformer}

Participating in message transfer to the operator, used in the optoelectronic system IM, it is implemented by means of the EOT. The formalized description of signal processing in IDS presented by the expression (6) allows us to analyze information transform in IDS from the system viewpoint as a whole and separately in each of its functional elements, including those in the display. Its analysis represents essential practical interest because of functional position of display in the system. Here, it is necessary to investigate principles of conversion of signals in optoelectronic IAE as well as regularities of their excitation by electrical signals. Creation of the analytical models considering specific properties of various types of these elements is important.

The formalized approach to signal processing in IDS allows to deduce the generalized operator expression (6) that describes symbol formation at IA. Analyzing the display from the information viewpoint, we can simplify this expression, presenting that optoelectronic system carries out data processing sequentially in four stages: 1) reception and the intermediate storage of the codes of messages, 2) their conversion according to IM, 3) formation of signals to excite IAE and 4) conversion of these electrical signals to the optical ones. Then, the expression (6) for synthesis of an arbitrary $v$-th message $S_{v}$ corresponding to the input code $C_{v}$ assumes the following form

$S_{v}=\psi_{\mathrm{S}}\left\{\psi_{\mathrm{E}}\left\{\psi_{\mathrm{D}}\left\{\psi_{\mathrm{T}}\left(C_{v}\right)\right\}\right\}\right\}$,

where $\psi_{\mathrm{S}}, \psi_{\mathrm{E}}, \psi_{\mathrm{D}}, \psi_{\mathrm{T}}$ are the operators describing conversion of the information from the electric form into the optical one, forming the signals to 
control the electrooptical transformer, conversion of codes in which it agrees IM and data storage, accordingly.

It is obvious that operator $\psi_{S}$ in the expression (7) presents information processing that is fulfilled by the display. Our analysis have shown that questions of synchronization of data streams, realizations of models of various types, constructions of drivers for EOT are enough explicitly described in the literature [3, 4, 5]. Analytical representation of digital information processing in these system elements is defined by the operators $\psi_{\mathrm{E}}, \psi_{\mathrm{D}}$ and $\psi_{\mathrm{T}}$. At the same time, conversion of messages from the electrical form into the optical one, which is described by the operator $\psi_{\mathrm{S}}$, is considered simplistically and based on representation of IAE in the form of quadripoles with an optical output [3].

In this approach, it is considered that exciting influence is brought to an input of a quadripole element and leads to appearance of an optical signal at its output. As a result, the appropriate element of a visual image of the messages is formed in IA [5]. Thus, it means that electrical signals applied to excitation of IAE completely correspond to the type of element, that is the generated input voltage of the converter possesses a required level and polarity, and the used current - necessary amplitude and direction. In some cases, this approach quite satisfies the tasks in view of the analysis of hardware for information display. It is quite reasonable if using individual or independent electrically connected IAEs. However, in modern devices in most cases it is necessary to control an EOT, elements of which are connected under the certain law, more often, in the form of a bi-dimensional matrix, or it is the same in a consecutive manner $[3,5]$.

Use of the simplified approach does not allow full formalization of the description of the operator $\psi_{S}$ that complicates the complex analysis of hardware realization in this group of devices and, accordingly, their optimization. Besides, in this case in the operator $\psi_{S}$ excitation features of various types of electrooptical elements are not considered. For example, presence of an output optical signal of the LED at the direct offset (current) and its absence at the reverse, or switching-on of a liquid-crystalline element at any polarity of the enclosed voltage, etc.

Interesting us information is transformed from the electrical form to the optical one which is described by the operator $\psi_{S}$ and corresponds to synthesis of an optical image of the $v$-th message $S_{v}$ from an electric signal $Z_{v}^{\mathrm{E}}$, which going on from the formula (7) can be presented as

$S_{v}=\psi_{\mathrm{S}}\left(Z_{v}^{\mathrm{E}}\right)$, where $Z_{v}^{\mathrm{E}}$ is an electrical signal that is brought to EOT, and $Z_{v}^{\mathrm{E}}=\psi_{\mathrm{E}}\left\{\psi_{\mathrm{D}}\left\{\psi_{\mathrm{T}}\left(C_{v}\right)\right\}\right\}$.

It is obvious that the signal $Z_{v}^{\mathrm{E}}$ necessary to excite EOT, is formed of the input code $C_{v}$. The basis for this conversion is logical signal processing in accord with IM electrical representation of the incoming message that is described by the operator $\psi_{\mathrm{D}}$. The generalized approach to realization of the functional conversion according to the expression (8) corresponds to the simplified representation of EOT in the form of the quadripole with an optical output.

From the functional viewpoint, data storage and formation of EOT control signals that are described by the operators $\psi_{\mathrm{T}}$ and $\psi_{\mathrm{E}}$ from the expression (7) are realized on the basis of the buffer converters coordinating separate stages of processing the messages by time and electrical parameters. Thus, as a rule, appropriate structural elements of devices do not participate in logical information processing. Therefore, revealing and researches of logical properties of an EOT is of the greatest practical interest. For the account and the analysis of these properties, we present the expression (8) in the following aspect

$S_{v}=\psi_{\mathrm{SOM}}\left\{\psi_{\mathrm{SLM}}\left(Z_{v}^{\mathrm{E}}\right)\right\}$,

where $\psi_{\text {SOM }}$ is the operator corresponding to multichannel conversion for representation of messages from the electrical form to the optical one; $\psi_{\mathrm{SLM}}$ - operator describing logical properties of an EOT.

It is obvious that $\psi_{\mathrm{SOM}}$ corresponds to a physical process in which the multi-channel information signal transferred by electrons turns to the multi-channel information signal transferred by photons. At the same time, $\psi_{\text {SLM }}$ is that other, as determination of a logical function of the structure with a number of inputs equal to the amount of inputs of an EOT, and the number of outputs corresponding to the amount of elements $p$ in it. For example, for the converter constructed on the basis of a bi-dimensional matrix $m \times n$ of the LEDs, $\psi_{\mathrm{SLM}}$ describes a logical structure with $m+n$ inputs and $p=m n$ outputs. This representation of EOT functioning is illustrated by Fig. 1 where serial conversion of an information signal $Z_{v}^{\mathrm{E}}$ by the unit of the logical processing that realizes the operator $\psi_{S L M}$ and the transformer of the physical form of the data corresponding to $\psi_{\text {SOM }}$ are shown. As a result, synthesized at the output of EOT is the optical image of an $v$-th message $S_{v}$. 


\section{Signal processing by a separate element of the display}

Let's consider formation of an optical image of a symbol $S_{v}$ from the set $\tilde{\boldsymbol{A}}_{v}$ of separate IAE, described by the formula (2), that is $S_{v} \Leftrightarrow \tilde{\boldsymbol{A}}_{v}$. Then, by analogy to the expression (9) for each element $a_{i_{v}} \in \tilde{\boldsymbol{A}}_{v}$, it is possible to write down

$a_{i_{v}}=\psi_{\mathrm{SO} 1}\left\{\psi_{\mathrm{SL} 1}\left(Z_{i}^{\mathrm{E}}\right)\right\}$,

where $a_{i_{v}}$ is the $i$-th element of an optical symbol $S_{v}$; $\psi_{\text {SO1 }}$ - operator corresponding to electrooptical transformation in an element of EOT; $\psi_{\mathrm{SL} 1}$ - logic function of this element; $Z_{i}^{\mathrm{E}}$ - signal of excitation of an element $a_{i_{v}} \in \tilde{\boldsymbol{A}}_{v}$, and $i=\overline{1, p}$.

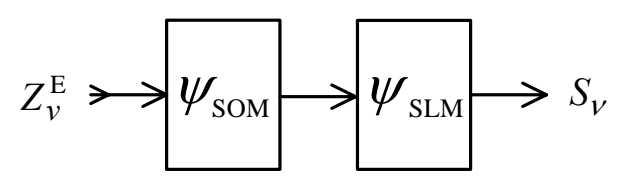

Fig. 1. Transformation of information from the electric form into the optical one in the case of account for logical properties of an electrooptical transformer.

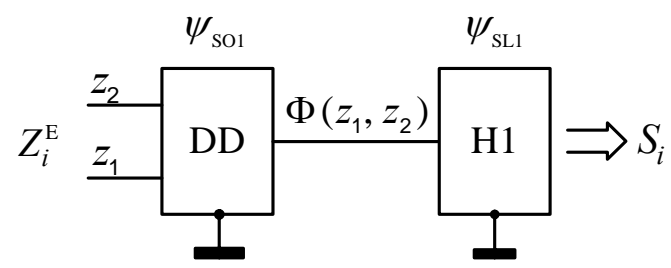

Fig. 2. Transformation of information from the electric form into the optical one in separate information area element taking into account its logical properties.

The functional analysis has shown that practically for all the types of IAE used in output units of means for display and information registration, the logic component $\psi_{\text {SL1 }}$ of realized conversion $\psi_{S}$ can be presented as a function of a logic gate. Features of this approach to the description of image synthesis at optoelectronic IA are illustrated in Fig. 2 where the functional diagram describing excitation of one IAE on the basis of the formula (10) is shown. Here, its electrical properties are presented in the form of a logic gate DD that realizes the function $\Phi\left(z_{1}, z_{2}\right)$ corresponding to the operator $\psi_{\mathrm{SL} 1}$. Electrooptical transformation is carried out by the element $\mathrm{H} 1$ according to the operator $\psi_{\mathrm{SO} 1}$. In the logical unit at the output of gate $\mathrm{DD}$, there is excitation $\mathrm{H} 1$, and $i$-th image element of the optical symbol $S_{v}$ is formed.
In the industrial optoelectronic IDS, there observed is wide application of various IAE that differ in their physical principles taken as a basics for electrooptical transformation of signals $[3,5]$. The greatest distribution in serial products is typical for IA based on liquid crystals, LEDs, electro- and cathodoluminescent radiators. Research of various types of elements has shown that at the logical approach to the excitation description they could be separated by two groups: polar and non-polar ones. Typical representatives of the first group are LED and cathodoluminescent displays. It is characteristic for them that for excitation it is necessary to submit opposite input signals to appropriate outputs. Liquid crystal cells, electroluminescent and vacuum heated radiators concern group of non-polar elements. In this case, excitation is realized at any connection of opposite signals to input wires.

Practical interest is related with definition of the logical function corresponding to the operator $\psi_{\text {SL1 }}$ from the expression (10). For this purpose, it is necessary to sequentially submit all possible combinations of logical signals to inputs of the investigated structure and to fix the received output response. Thus, we generate the truth table of a logic gate and, as a result, we can define its type [6, 7].

The analysis of response of the structure represented in Fig. 2 on the full group of combinations of input signals allows to establish the function $\Phi\left(z_{1}, z_{2}\right)$ of element DD with which help the logical properties shown at excitation IAE are modeled. For polar elements, it was accepted that the anode and cathode of the LED are its first and second input. And so, input signals $z_{1}$ and $z_{2}$ are brought to them, accordingly. For cathodoluminescent elements, it corresponds to the anode (or to a controlling electrode a grid) and to the cell cathode.

Investigation of the polar IAE, results of which are presented in Table 1, have shown that the operator $\psi_{\text {SL1 }}$ realizes logical function $\Phi_{p}$ “ $z_{1}$ does not imply $\mathrm{z}_{2}$ " ("true if $\mathrm{z}_{1}$ but not $\mathrm{z}_{2}$ "). It concerns the group of logical implication functions. In hardware realization, they are not usually implemented as elementary circuits, but rather as combinations of a gate with an inverter at one input/output. The logical operator $\psi_{\text {SL1 }}$ uses negation of implication $\Phi_{p}$, which in the Boolean algebra is possible to be presented as $\psi_{\mathrm{SL} 1}\left(Z_{i}^{\mathrm{E}}\right) \Leftrightarrow \Phi_{p}=z_{2} \not \subset z_{1}=\overline{\overline{z_{1}}+z_{2}}$.

Accordingly, proceeding from the expressions (10) and (11), excitation of arbitrary polar IAE $\tilde{a}_{p i_{v}}$ can be presented in the following aspect

$$
\tilde{a}_{p i_{v}}=\psi_{\mathrm{SO} 1}\left\{\overline{\overline{z_{1}}+z_{2}}\right\} \text {. }
$$


Table 1. Research of logical properties of polar information area elements.

\begin{tabular}{|c|c|c|c|c|}
\hline \multicolumn{2}{|c|}{$\begin{array}{c}\text { Input } \\
\text { logical signals }\end{array}$} & $\begin{array}{c}\text { Output } \\
\text { logical signal }\end{array}$ & $\begin{array}{c}\text { Output } \\
\text { optical } \\
\text { signal }\end{array}$ & $\begin{array}{c}\text { Logical } \\
\text { function } \\
\text { of the element }\end{array}$ \\
\hline$z_{1}$ & $z_{2}$ & $\Phi_{p}\left(z_{1}, z_{2}\right)$ & $\tilde{a}_{p i_{v}}$ & $\psi_{\mathrm{SL} 1}$ \\
\hline 0 & 0 & 0 & no & \multirow{4}{*}{$z_{2} \not \subset z_{1}$} \\
\hline 0 & 1 & 0 & no & \\
\hline 1 & 0 & 1 & yes & \\
\hline 1 & 1 & 0 & no & \\
\hline
\end{tabular}

Using this expression, it is possible to offer a logical model of polar IAE, depicted in Fig. 3. The model uses three logical elements that realize two functions of the Boolean basis: two inverters D1, D3 and OR gate D2. The element $\mathrm{H} 1$ carries out electrooptical transformation and forms an image element in the case of truth logical value at the output of D3 gate.

Our analysis of the non-polar IAE, results of which are summarized in Table 2, has shown that the operator $\psi_{\text {SL1 }}$ realizes the logical function $\Phi_{n p}$ exclusive or ("one or the other but not both"). It represents the inequality function that sometimes is named as the addition modulo 2. In hardware realization, it is usually implemented as an elementary XOR gate. So, in this case the logical operator $\psi_{\mathrm{SL} 1}$ uses the inequality function $\Phi_{n p}$ that in the Boolean algebra can be

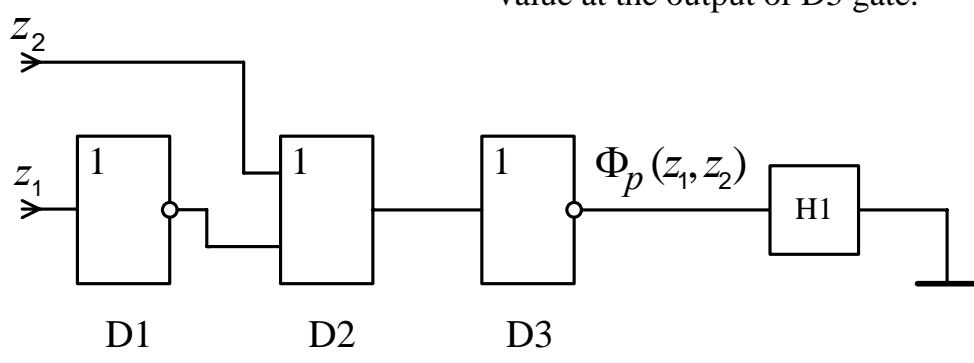

represented as

$$
\begin{aligned}
& \psi_{\mathrm{SL} 1}\left(Z_{i}^{\mathrm{E}}\right) \Leftrightarrow \Phi_{n p}= \\
& =z_{1} \oplus z_{2}=z_{1} \cdot \overline{z_{2}}+\overline{z_{1}} \cdot z_{2} .
\end{aligned}
$$

Table 2. Research of logical properties of non-polar information area elements.

\begin{tabular}{|c|c|c|c|c|}
\hline \multicolumn{2}{|c|}{$\begin{array}{c}\text { Input } \\
\text { logical signals }\end{array}$} & $\begin{array}{c}\text { Output } \\
\text { logical signal }\end{array}$ & $\begin{array}{c}\text { Output } \\
\text { optical } \\
\text { signal }\end{array}$ & $\begin{array}{c}\text { Logical } \\
\text { function } \\
\text { of the element }\end{array}$ \\
\hline$z_{1}$ & $z_{2}$ & $\Phi_{n p}\left(z_{1}, z_{2}\right)$ & $\tilde{a}_{n p i_{v}}$ & \multirow{2}{*}{$\psi_{\text {SL1 }}$} \\
\hline 0 & 0 & 0 & no & \multirow{2}{*}{$z_{1} \oplus z_{2}$} \\
\hline 0 & 1 & 1 & yes & \\
\hline 1 & 0 & 1 & yes & \\
\hline 1 & 1 & 0 & no & \\
\hline
\end{tabular}

Therefore, going on from the expressions (10) and (11), it is possible to present excitation of an arbitrary non-polar IAE $\tilde{a}_{n p i_{v}}$ in the following form

$$
\tilde{a}_{n p i_{v}}=\psi_{\text {SO1 }}\left\{z_{1} \cdot \overline{z_{2}}+\overline{z_{1}} \cdot z_{2}\right\} \text {. }
$$

Using this expression, it is possible to offer a logical model of non-polar IAE, depicted in Fig. 4. The model uses five logical elements that realize all three functions of the Boolean basis: two inverters (D1, D2), two AND gates (D3, D4) and one OR gate D5. The element $\mathrm{H} 1$ carries out electrooptical transformation and forms an image element in the case of a truth logical value at the output of D5 gate.

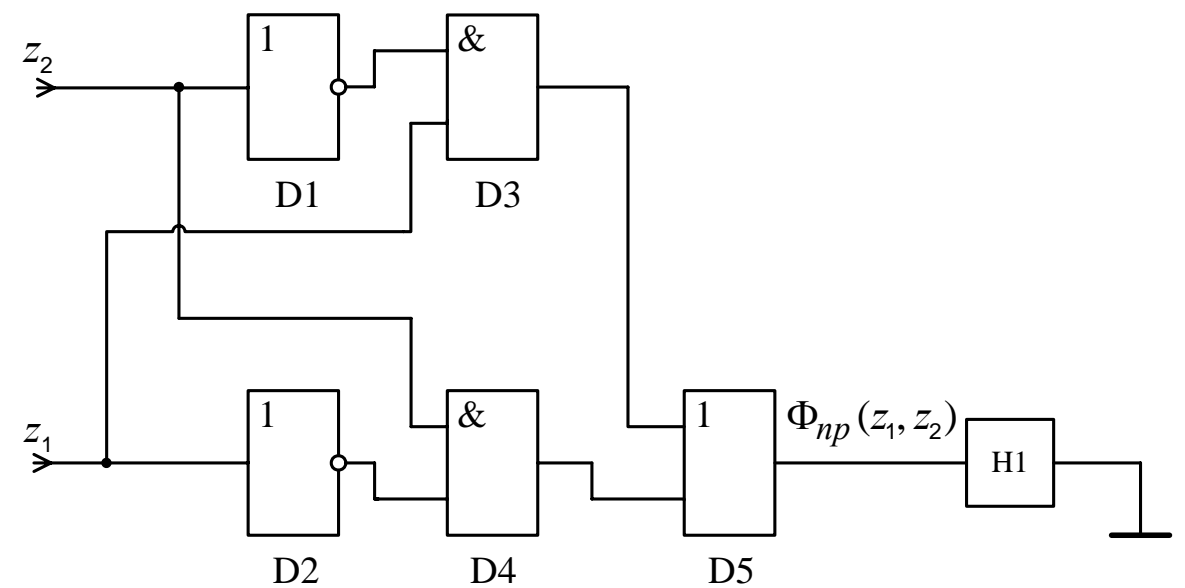

Fig. 4. Logical Boolean model of a non-polar information area element. 


\section{Conclusion}

We have offered the generalized analytical description of IDS based on the functional approach to information processing in the human-machine environment. When researching the basic attention was given to the least investigated question of logic properties of a separate element of the display. Based on the analysis of the functional properties, we selected groups of polar and non-polar electrooptical transformers. It has been shown that polar IAE (LEDs, cathodoluminescent displays, etc.) realize the logical function of negation of implication. At the same time, non-polar IAE (liquid crystal cells, electroluminescent and vacuum heated radiators) transform the data according to the inequality function. Furthermore, we present analytical models of logical information processing by polar and non-polar elements of displays. Moreover, given are the equivalent circuits in the Boolean basis that can replace polar and non-polar IAE at logical functional modeling of display units.

The obtained results allow formalizing the description and modeling of IDS both in whole and in their separate units by logical representation of appropriate functions. It forms an analytical basis for complex optimization and hardware minimization of technical decisions in optoelectronic IDS, as well as essential increase in their reliability.

\section{References}

1. W. Marras, W. Karwowski. The occupational ergonomics handbook. Interventions, controls, and applications in occupational ergonomics. - Boca Raton: CRC/Taylor \& Francis, 2006.

2. W. Karwowski. Handbook on standards and guidelines in ergonomics and human factors. Mahwah, N.J.: Lawrence Erlbaum Associates, 2006.

3. Xun Li. Optoelectronic Devices: Design, Modeling, and Simulation. - Cambridge University Press, 2009.

4. M. Parker. Physics of Optoelectronics. - CRC Press, 2005.

5. S. Kasap. Optoelectronics and Photonics: Principles and Practices. - Prentice Hall, 2001.

6. R. Jaeger. Microelectronic circuit design. McGraw-Hill, 1997.

7. J. Bird. Engineering Mathematics. - Elsevier Science \& Technology, 2010. 